\title{
ON DESIGN OF SELF-TUNING ACTIVE FILTERS
}

\author{
UDC ((621.372.54:004.382+621.3.049.77):005.334)
}

\author{
Darko Mitić, Goran Jovanović, Mile Stojčev, Dragan Antić \\ University of Niš, Faculty of Electronic Engineering, Niš, Republic of Serbia
}

\begin{abstract}
In this paper, we present one approach in design of self-tuning all-pass, bandpass, low-pass and notch filters based on phase control loops with voltage-controlled active components and analyze their stability as well. The main idea is to vary signal delay of the filter and in this way to achieve phase correction. The filter phase characteristics are tuned by varying the transconductance of the operational transconductance amplifier or capacitance of an MOS varicap element, which are the constituents of filters. This approach allows us to implement active filters with capacitance values of order of $p F$, making the complete filter circuit to be amenable for realization in CMOS technology. The phase control loops are characterized by good controllable delay over the full range of phase and frequency regulation, high stability, and short settling (locking) time. The proposed circuits are suitable for implementation as a basic building RF function block, used in phase and frequency regulation, frequency synthesis, clock generation recovery, filtering, selective amplifying etc.
\end{abstract}

Key words: all-pass filter, band-pass filter, low-pass filter, notch filter, low-noise amplifier, operational transconductance amplifier, phase synchronizer, channel selector, phase control loop, stability analysis

\section{INTRODUCTION}

Continuous-time filters realized by using operational transconductance amplifiers (OTAs) and capacitors, known also as $g_{m}-C$ or OTA-C filters, are basic building blocks in analog signal processing. Applications of these filters involve frequency of operation from a few tens of hertz up to gigahertz [1]. OTA-C filters offer many advantages over other continuous-time filters in terms of low power and high frequency capability [2]. They are used for applications such as selective amplifiers, IF and RF filters [1-4], filters for high-speed baseband data links [5], equalizers for bit detectors in magnetic and optical disk recording [5], medical electronics [6, 7], video signal processing [8], phase synchronizers [9], image-rejection in super-heterodyne receiver [10], etc.

Received May 04, 2020

Corresponding author: Darko Mitić

University of Niš, Faculty of Electronic Engineering, Aleksandra Medvedeva 14, 18000 Niš, Republic of Serbia

E-mail: darko.mitic@elfak.ni.ac.rs 
An inherent problem in implementing analog filters is that the values of manufactured analog circuit components often differ from the design specifications because of process parameters, supply voltage, and temperature (PVT) variations. Due to these perturbations, the practically obtained results are not optimal. It is, therefore, necessary to carefully test all analog integrated circuits (ICs) and to discard any those that do not meet the specifications. Another way to compensate the influence of PVT variations to frequency characteristics are based on utilization of tunable filters, master-slave filter tuning schemes and self-tuning filters. The overview of the authors' recent results [11-14] in design of self-tuning filters is presented in this paper.

Tunable filters are realized by using digitally controlled binary-weighted capacitor array, or current mirror array [8]. The switching scheme is based on successive approximation algorithm. However, the frequency accuracy with digital tuning is constrained by the number of tuning (not larger than 5) bits used.

Analog filters are also tuned by master-slave tuning schemes [2]. The most commonly used master-circuit is voltage-controlled oscillator (VCO), whereas a slave filter is built with identical integrators. However, there are some difficulties in matching filter characteristics with characteristics of the master VCO [15]. Superior approach is to use voltage-controlled filter as a master what results in a better matching between master and slave filters.

Each filter at characteristic frequencies (cut-off, central frequency etc.) has defined phase shifts values. By comparing the phases of input and output signals, it is possible to detect the mismatch of filter characteristics. In order to obtain the desired phase transfer function, self-tuning filters use the estimated phase error as the correction factor. In our proposal, to accomplish the phase error correction we use a phase control loop, similar to one used in tuning oscillators with the phase locked loop (PLL) or delay lines with the delay locked loop (DLL) [16-18].

Typically, DLL is a feedback control system that attempts to maintain a constant or a zero phase difference between two signals. In DLL, the input signal passes through a chain of delay elements, forming a voltage controlled delay line (VCDL). The amount of delay depends on the phase difference between input and output VCDL signals. A steady state is reached when these two signals are synchronized [18]. There are several different methods for implementing VCDL. VCDL can be classified as analogue and digital. In the analogue implementation, the delay element is realized by an active filter [11]. The inverter is used as the delay cell in the digital realization of $\operatorname{VCDL}[19,20]$. Each of these methods has its advantages and drawbacks. Digitally realized VCDLs are designed only for processing digital clock signals. However, it is not possible to achieve phase synchronization between two sinusoidal signals by using the digital DLL circuit. That is why the solutions given in $[11,12,14]$ and $[13]$ are considered, where VCDLs are substituted by voltage-controlled active filters (VCAF) and low-noise amplifier, respectively. This approach provides the phase correction of sinusoidal or some other complex periodical signals without violating the other characteristics of the input signal.

The goal of this paper is to present the main authors' results in design of self-tuning allpass, band-pass and notch filters by using phase control loops with voltage-controlled $g_{m}-C$ active filters $[11,12,14]$. It is also shown how a band-pass filter can be realized with a lownoise amplifier by using the same control approach and MOS varicap as a voltage-controlled element instead of OTA [13]. The filters' stability is considered thoroughly. Last but not least, it is demonstrated how results presented in [12] can be used in the synthesis of low- 
pass active filters. In comparison to a DLL circuit, a delay correction becomes now a phase correction while all good properties of DLL, such as the first-order loop, high stability and short settling time (i.e. low latency resulting in high system throughput), are preserved.

The rest of the paper is organized as follows. In Section 2, the novel phase control loop principle of operation is described. Section 3 presents basic elements of the proposed phase control loop. The self-tuning all-pass filter, obtained by substituting of voltage-controlled active filter (see Fig. 2) with all-pass filter chain, resulting in phase synchronizer [11], is presented in Section 4. It is shown in Section 5 that when a band-pass filter is used as an active filter, the proposed circuit becomes a self-tuning band-pass filter, also acting as a selective amplifier [12]. The design of a self-tuning band-pass filter by using low-noise amplifier with MOS varicap element instead of OTA [13] is discussed in Section 6. The approach given in Section 5 can be modified to serve as a channel selector if the low-pass component of the band-pass filter is used as the voltage-controlled active filter, resulting in a self-tuning low-pass filter elaborated in Section 7. Finally, the design of a self-tuning notch filter [14] is described in Section 8.

\section{OPERATION PRINCIPLE OF SELF-TUNING ACTIVE FILTERS}

The proposed self-tuning active filters are based on the phase control loop providing the phase control in similar manner as a DLL circuit [16-18] does, with one exception: instead of the pulse delay control as it is in a DLL circuit, the phase of analogue output signal is tuned [21]. The block diagrams of the classical DLL circuit and the proposed novel phase control loop are presented in Fig. 1 and Fig. 2, respectively. VCDL as a constituent of a DLL circuit (Fig. 1) is substituted by VCAF in Fig. 2. In this way, the phase of $V_{\text {out }}$ can be adjusted in a wide range of regulations by implementing a phase loop control method [11-14]. Zero crossing detectors are introduced to amplify and to process the analogue signals for a phase detector. Other constituents of phase control loops (phase detector, charge pump and low-pass filter) have similar structures as in DLL circuit, and they will be all described further in the next section.

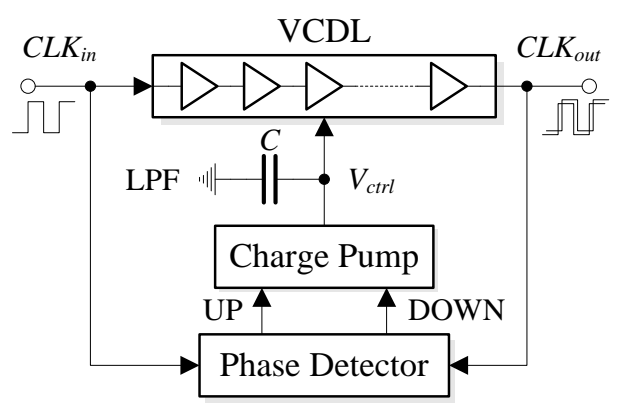

Fig. 1 Block diagram of DLL 


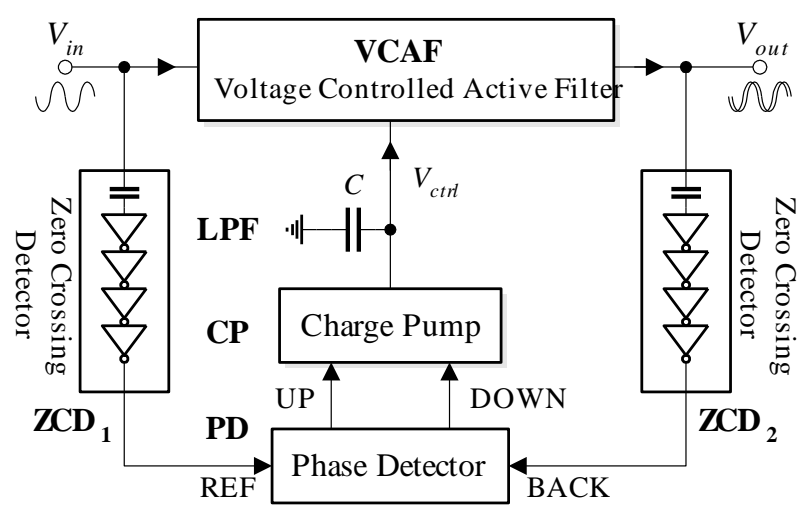

Fig. 2 Block diagram of proposed self-tuning active filter

\section{DESCRIPTION OF PHASE CONTROL LOOP ELEMENTS}

The design of voltage-controlled filters, presented in this paper, is done by using either an OTA or a MOS varicap element (MVaricap).

OTA is a voltage controlled current source. It is widely used as an essential element of transconductance capacitor filters, $\Delta \Sigma$ modulators, gyrators, variable gain amplifiers, and negative-resistance elements. The use of circuits composed of OTA and capacitors has its advantages in the synthesis of monolithic analogue building blocks, because of its ability of constructing active filters with small capacitance value (order of $\mathrm{pF}$ ), in comparison to the conventional active filters techniques, where capacitance values are of order of several $\mathrm{nFs}$ for the same frequency range. Thanks to the small capacitance values, the complete filter circuit can be realized on the single VLSI CMOS chip [22, 23].

The filter phase characteristic is tuned by varying the transconductance $g_{m}$ of OTA. The structure of OTA is presented in Fig. 3. The transconductance $g_{m d}$ of the differential stage is given by:

$$
g_{m d}=\sqrt{\mu C_{o x}\left(\frac{W}{L}\right)_{M_{1}, M_{2}} I_{s s}},
$$

where $I_{S S}$ is:

$$
I_{s s}=\frac{\mu C_{o x}}{2}\left(\frac{W}{L}\right)_{M_{3}}\left(V_{b i a s}-V_{t h}\right)^{2},
$$

and $\mu$ is a charge-carrier effective mobility, $C_{o x}$ is a gate oxide capacitance per unit area; $W$ is a gate width, $L$ is a gate length, $V_{t h}$ is a threshold voltage, and $V_{\text {bias }}$ is a control voltage. For more details, related to Equations (1) and (2), someone can refer to [24]. The usage of OTA with voltage-controlled transconductance, contrary to the traditional approach (OTA with constant $g_{m}$ ), enables us to realize a voltage-controlled $g_{m}-C$ filters $[3,8,25-27]$. 


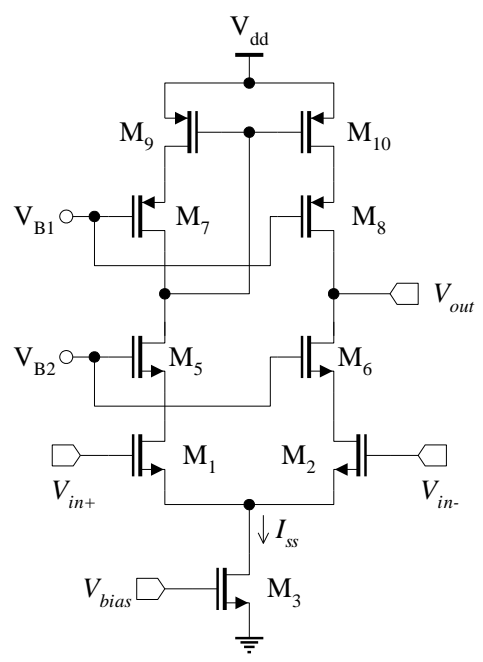

Fig. 3 Schematics of OTA [12]

MOS varicap element corresponds to the voltage controlled capacitor. In IHP BiCMOS technology, it is implemented as a modified PMOS transistor [28]. By controlling polarization voltage of a N-well, the capacitance between gate and channel of the PMOS transistor varies. The value of a control voltage, $V_{\text {ctrl }}$, determines a capacitance of the MOS varicap element [28]:

$$
C_{\text {MVaricap }}=\frac{\bar{C}_{\text {MVaricap }}}{\sqrt{1+V_{\text {crrl }} / V_{\rho}}},
$$

where $\bar{C}_{\text {MVaricap }}$ and $V_{\rho}$ denote model parameters. MOS varicap element is implemented in the design of the voltage-controlled low-noise amplifier used to amplify a very weak received signal and, therefore, considered as one type of band-pass filter.

A non-inverting operational amplifier (OA) with unity gain (Fig. 4) is also implemented to reduce the influence of the load impedance $Z_{L}$ on the filter transfer function or to decouple the influence of $C_{L P F}$ to MOS varicap capacitance.

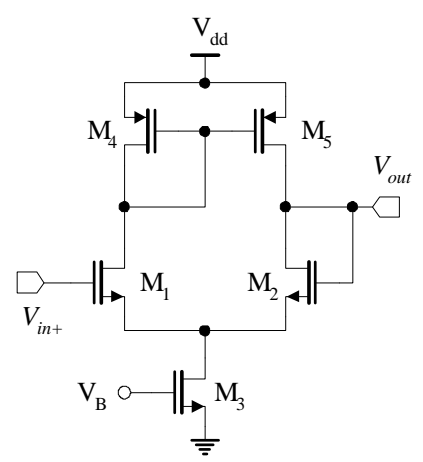

Fig. 4 Schematics of OA [11] 
Prior to detecting the phase shift between analogue signals $V_{\text {out }}$ and $V_{\text {in }}$, these signals are amplified and converted to a rectangular form by the zero crossing detector (ZCD) [11] depicted in Fig. 5. ZCD consists of four CMOS inverter stages, where the first one, $\mathrm{A}_{1}$, acting as a linear amplifier, is capacitive coupled with the analog signal obtained from the voltage-controlled filter. Therefore, $\mathrm{A}_{1}$ eliminates $\mathrm{DC}$ offset while the other three stages, $\mathrm{A}_{2}, \mathrm{~A}_{3}$ and $\mathrm{A}_{4}$, operate as digital inverter ones.

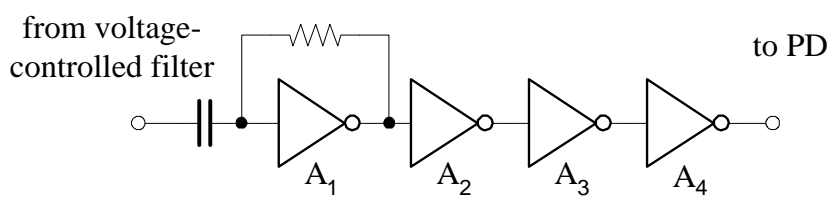

Fig. 5. Zero crossing detector

The schematic of a phase detector (PD) is shown in Fig. 6(a) [11]. It estimates the phase difference between the reference signal REF, the output of $\mathrm{ZCD}_{1}$ (see Figure 2), and the signal BACK, the output of $\mathrm{ZCD}_{2}$, and generates rectangular signals UP and DOWN whose widths are proportional to the phase error. Its hardware structure is adopted from [17]. The PD operation principle is depicted in Fig. 6(b). UP (DOWN) signal is active when the phase of the REF (BACK) signal leads in respect to the phase of the BACK (REF) signal. Waveforms on the left side of Fig. 6(b) correspond to the case when the BACK signal leads in respect to the REF signal. Diagrams on the right side are valid when the REF signal leads in respect to the BACK signal.

The structure of the charge pump (CP) is depicted in Fig. 7. It is loaded by the capacitor $C_{L P F}$ representing a loop filter. Transistors $\mathrm{M}_{1}$ and $\mathrm{M}_{4}$ are driven by UP and DOWN pulses and act as switching elements, enabling charging and discharging of $C_{L P F}$, whereas transistors $\mathrm{M}_{2}$ and $\mathrm{M}_{3}$ are employed as the current sink and source, respectively. The control voltage $V_{\text {ctrl }}$ drives VCAF and can be written as:

$$
V_{c t r l}=-k_{c p} \int_{-\infty}^{t} \theta \mathrm{d} \tau,
$$

where $k_{c p}$ is a positive CP parameter:

$$
k_{c p}=\frac{I_{c p}}{C_{L P F}},
$$

depending on $I_{C P}$ (constant current of a charge pump, used for charging or discharging $C_{L P F}-$ see Fig. 7) and the capacitor $C_{L P F}$. Thanks to the small value of $I_{C P}$, the phase loop capacitor $C_{L P F}$ could be implemented directly on-chip [11].

The bias circuit is intended to provide correct $\mathrm{CP}$ operation. This block generates two bias voltages, $V_{\text {bias_ } P}$ and $V_{\text {bias } N}$. More design details, which relate to the bias circuit, are given in [18]. The control voltages, $V_{\text {bias_ }} P$ and $V_{\text {bias }} N$, define charging and discharging currents of $C_{L P F}$. 


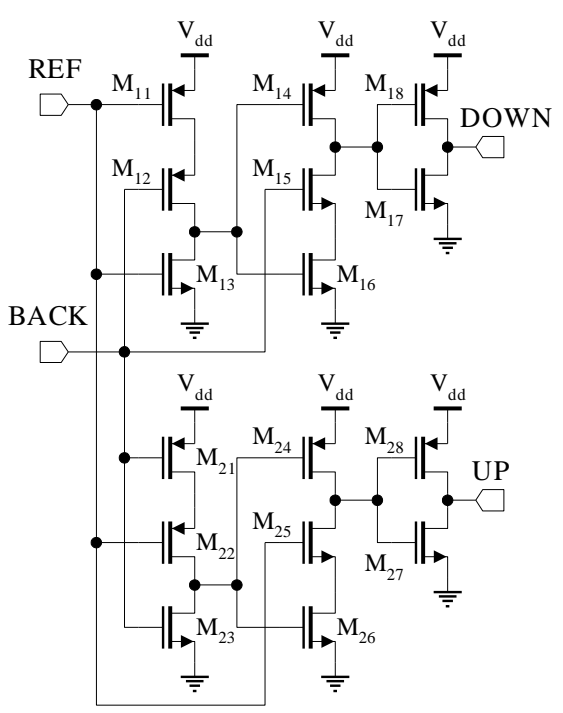

(a)
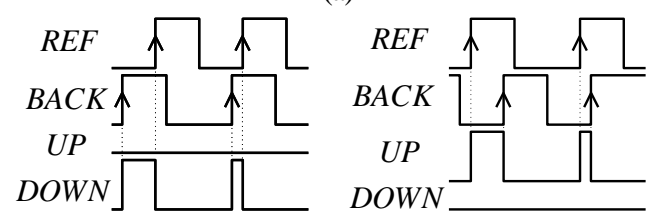

(b)

Fig. 6 Schematic of phase detector (a) and signals waveforms (b) [11]

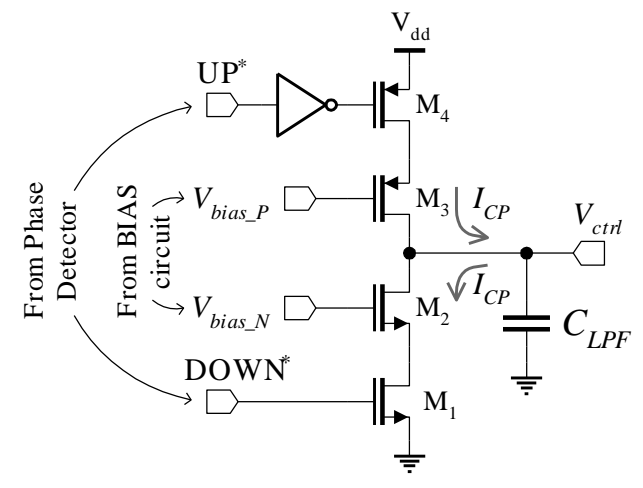

Fig. 7 Charge pump with phase loop filter [12] 


\section{SELF-Tuning All-PASs FILTER}

In this implementation, VCAF is implemented as the chain of $g_{m}-C$ all-pass filters [11], which have no influence on signal amplitude, whereas the phase of the analogue signal could be varied $[21,29,30]$. Such self-tuning filter is shown in Fig. 8 and can be used then as a phase-synchronizer.

\section{VCAPF}

Voltage Controlled All-Pass Filters

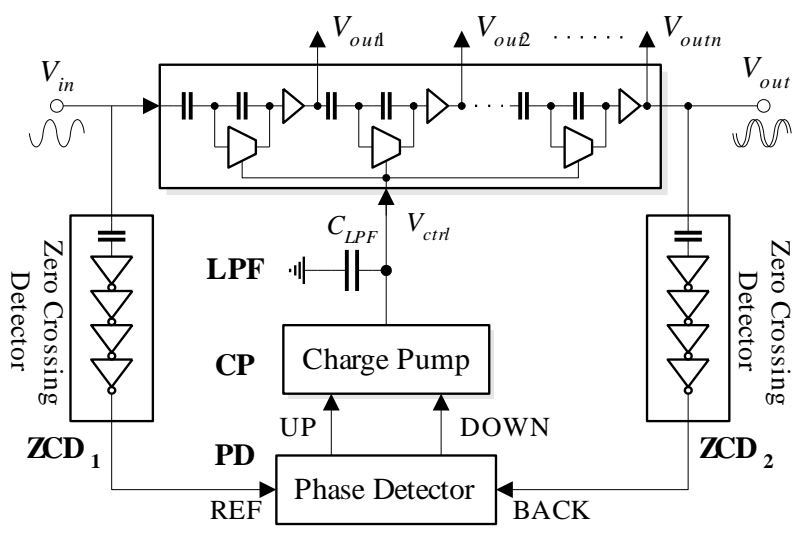

Fig. 8 Self-tuning all-pass filter [11]

A scheme of one first-order all-pass filter is given in Fig. 9(a). The all-pass filter is realized as a continuous $g_{m}-C$ filter [29]. By substituting OTA with transconductance $g_{m}$, we obtain the equivalent electrical model presented in Fig. 9(b). Since OA has no influence on the transfer characteristic of the filter, it is omitted in the equivalent electrical scheme.
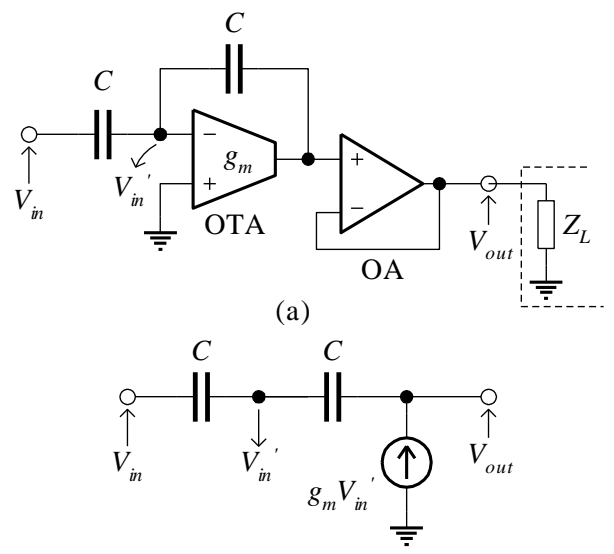

(b)

Fig. 9 All-pass filter: electrical scheme (a) and model (b) [11] 
The transfer function of all-pass filter is:

$$
H(s)=\frac{V_{\text {out }}}{V_{\text {in }}}=\frac{s-\frac{g_{m}}{C}}{s+\frac{g_{m}}{C}} .
$$

Its amplitude characteristic is given by:

and the phase characteristic is:

$$
|H(\omega)|=1
$$

$$
\theta(\omega)=-\pi-2 \arctan \left(\frac{\omega C}{g_{m}}\right)
$$

The phase characteristic is determined in the term of admittance $\omega C$ and amplifier transconductance $g_{m}$. As we have already said, this characteristic would be tuned by using OTA transconductance $g_{m}$, which is a better and efficient solution from the aspect of CMOS technology.

The phase of an $n$-stage voltage-controlled all-pass filter chain is defined by:

$$
\theta_{A P}=-n \pi-2 \cdot n \cdot \arctan \left(\frac{\omega C}{g_{m}}\right),
$$

with $g_{m}$ determined as:

$$
g_{m}=g_{m d}+g_{m c}
$$

where $g_{m c}=$ const. (for more details refer to [11]) and:

$$
g_{m d}=k_{m d}\left(V_{c t r l}-V_{t h}\right),
$$

with $V_{c t r l}$ corresponding to $V_{\text {bias }}$ in (2) and:

$$
k_{m d}=\mu C_{o x} \sqrt{\frac{1}{2}\left(\frac{W}{L}\right)_{M_{1}}\left(\frac{W}{L}\right)_{M_{2}, M_{3}}},
$$

from (1) and (2), considering that $V_{\text {bias }}=V_{c t r l}$.

The phase shift of the output signal for the $i$-th stage is given by

$$
\theta_{A P}^{i}=-i \pi-2 \cdot i \cdot \arctan \left(\frac{\omega C}{g_{m}}\right), \quad 1 \leq i \leq n,
$$

and multi-phase signals, $V_{\text {out } 1}, \ldots, V_{\text {outn }}$ are generated at the outputs of each all-pass filter in the chain. The stability issue of the proposed solution is discussed by proving the following theorem [11]. 
Theorem 1: The phase control loop with the all-pass filter chain, defined by (9), (10) and (11), with the control (4), where $\theta=\theta_{A P}$, is globally asymptotically stable implying that $\theta_{A P} \rightarrow 0$ for any initial non-zero value of the phase shift.

Proof: The phase loop dynamics is directly obtained by time-differentiation of (9):

$$
\frac{\mathrm{d} \theta_{A P}}{\mathrm{~d} t}=-\frac{2 n k_{c p} k_{m d} \omega C}{\left(k_{m d}\left(V_{c t r l}-V_{t h}\right)+g_{m c}\right)^{2}+(\omega C)^{2}} \theta_{A P},
$$

taking into account (8), (10) and (11).

In order to prove that the phase dynamics (14) is stable, we will use the Lyapunov's stability theorem [31]. The Lyapunov's function candidate $E\left(\theta_{A P}\right)$ is chosen to be:

$$
E\left(\theta_{A P}\right)=\frac{1}{2} \theta_{A P}^{2} .
$$

As we can see, it is the positive definite function $E\left(\theta_{A P}\right)>0$ for $\forall \theta_{A P} \neq 0, E\left(\theta_{A P}\right)=0$ for $\theta_{A P}=0$ and $E\left(\theta_{A P}\right) \rightarrow \infty$ when $\theta_{A P} \rightarrow \infty$. Since its time-derivative:

$$
\frac{\mathrm{d} E\left(\theta_{A P}\right)}{\mathrm{d} t}=-\frac{2 n k_{c p} k_{m d} \omega C}{\left(k_{m d}\left(V_{c t r l}-V_{t h}\right)+g_{m c}\right)^{2}+(\omega C)^{2}} \theta_{A P}^{2}<0
$$

is the negative definite function for $\forall \theta_{A P} \neq 0$, according to the Lyapunov's stability theorem [31], one can conclude that the equilibrium point of (14) $\theta_{A P}=0$ and, consequently, the overall system are globally asymptotically stable, i.e. the phase $\theta_{A P}$ tends to zero asymptotically from any initial non-zero value.

OTA is implemented in IHP $0.25 \mu \mathrm{m}$ SiGe BiCMOS technology, with the supply voltage $V_{d d}=3 \mathrm{~V}$ [32]. In the middle of a regulation range, with $V_{\text {bias }}=0.7 \mathrm{~V}$, OTA has the first pole at $f_{p}=1.1 \mathrm{MHz}$, gain-bandwidth product $\mathrm{GBW}=600 \mathrm{MHz}$, open loop gain $A_{0}=62.5 \mathrm{~dB}$, and output resistance $R_{\text {out }}=2.4 \mathrm{M} \Omega$.

Fig. 10 depicts the time response of the phase-synchronizer (power-on sequence). Waveforms of input signal $V_{\text {in }}$ and output $V_{\text {out }}$ for the four stage all-pass filter chain ( $n=$ 4) are presented in Fig. 10(a). The frequency of the input signal is $f=50 \mathrm{MHz}$. The waveforms of UP and DOWN signals, obtained at the outputs of PD, as well as $V_{c t r l}$, are given in Fig. 10(b). The steady-state state is reached, i.e. the phase loop is locked, at the moment when: $i$ ) the phases of signals $V_{i n}$ and $V_{\text {out }}$ are equal, $\left.i i\right)$ UP and DOWN signals disappear, and iii) the control voltage $V_{c t r l}$ has a constant value. The settling time is approximately $300 \mathrm{~ns}$. 

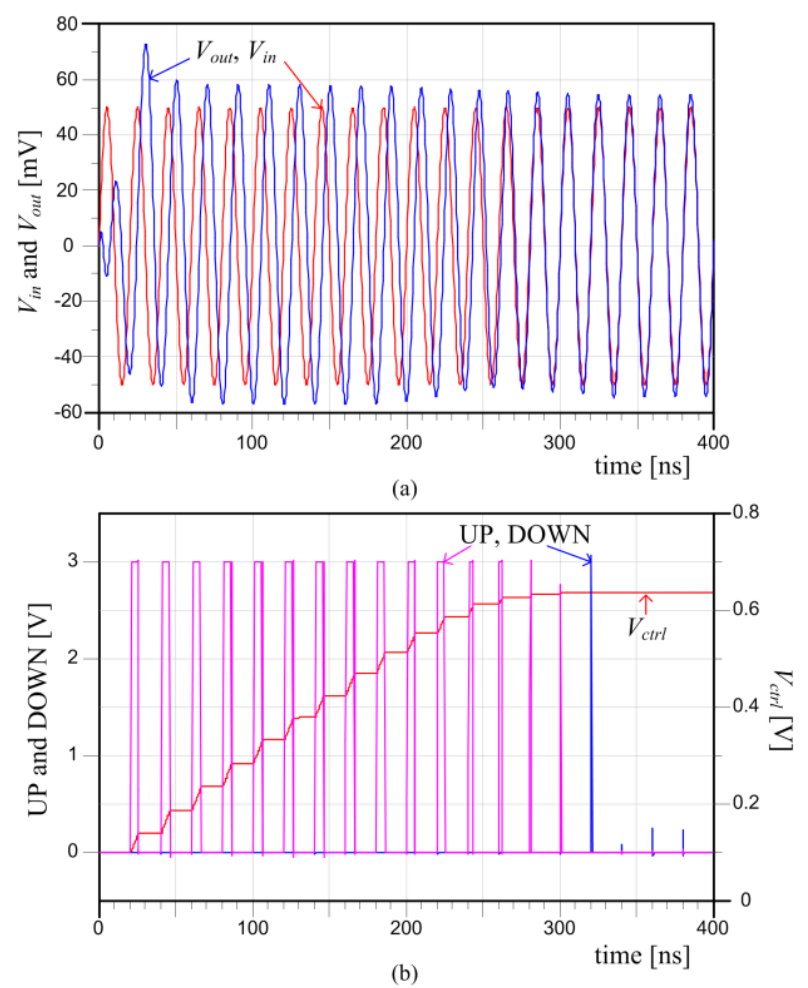

Fig. 10 Time response of self-tuning all-pass filter chain: (a) $V_{\text {in }}$ and $V_{\text {out }}$ and (b) UP, DOWN and $V_{c t r l}[11]$

\section{SELF-TUNING BiQuAd BAND-PASS FILTER}

The proposed phase control loop can be used in the design of the self-tuning $g_{m}-C$ biquad band-pass filter [12]. It forces the filter central frequency to be equal to the input signal frequency by adjusting OTA transconductance $g_{m}$. Thanks to that, the filter can be also used as a selective amplifier. The self-tuning biquad band-pass filter is shown in Fig. 11.

In this implementation, a voltage-controlled biquad band-pass filter is utilized as VCAF and it is marked by VCBPF in Fig. 11. It consists of two low-pass filters (LPFs), in an ideal case of two integrators, connected to form a closed loop system. The first LPF is located in the forward path, whereas the second one is placed in the feedback. LPFs are realized with $\mathrm{OTA}_{1}, \mathrm{OTA}_{2}$ and capacitors $\left(C_{1}\right.$ and $\left.C_{2}\right)$. In order to determine the transfer function of $g_{m}-C$ biquad band-pass filter, the equivalent model, depicted in Fig. 12, is used. As it is well known, real OTA has finite output resistance. The model, given in Fig. 12 , includes resistors $R_{01}$ and $R_{02}$ that correspond to OTA $\mathrm{A}_{1}$ and OTA $\mathrm{O}_{2}$ output resistances, respectively. 


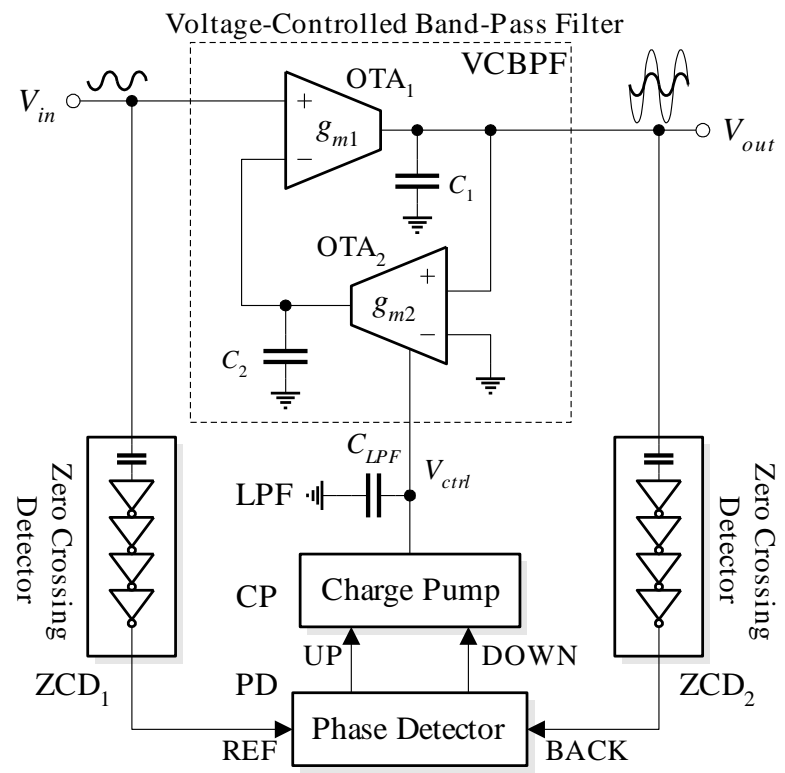

Fig. 11 Self-tuning biquad band-pass filter [12]

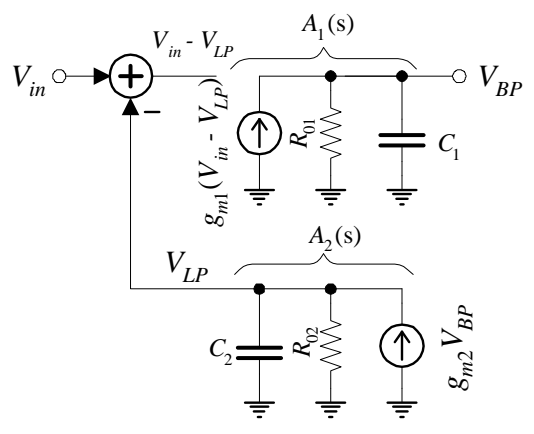

Fig. 12 Equivalent model of biquad band-pass filter (VCBPF) [12]

The LPF transfer functions are given by:

$$
A_{1}(s)=\frac{g_{m 1} R_{01}}{1+s C_{1} R_{01}}=\frac{A_{01}}{1+\frac{s}{\omega_{1}}}=\frac{\omega_{1} A_{01}}{s+\omega_{1}}
$$

and

$$
A_{2}(s)=\frac{g_{m 2} R_{02}}{1+s C_{2} R_{02}}=\frac{A_{02}}{1+\frac{s}{\omega_{2}}}=\frac{\omega_{2} A_{02}}{s+\omega_{2}},
$$

where $A_{01}=g_{m 1} R_{01}$ and $A_{02}=g_{m 2} R_{02}$ are Bode gains, and $\omega_{1}=2 \pi f_{1}=1 /\left(C_{1} R_{01}\right)$ and $\omega_{2}=2 \pi f_{2}=1 /\left(C_{2} R_{02}\right)$ are the poles of LPFs. 
The closed loop transfer function of the circuit presented in Fig. 8(a) corresponds to the following biquad band-pass filter transfer function:

$$
H_{B P}(s)=\frac{V_{B P}}{V_{\text {in }}}=\frac{A_{1}(s)}{1+A_{1}(s) \cdot A_{2}(s)}=\frac{A_{01} \omega_{1}\left(s+\omega_{2}\right)}{s^{2}+s\left(\omega_{1}+\omega_{2}\right)+\left(1+A_{01} \cdot A_{02}\right) \cdot \omega_{1} \cdot \omega_{2}} .
$$

Assuming that $A_{01}, A_{02}>1$ and that the pole $\omega_{1}$ of the first LPF is a dominant one, whereas the pole $\omega_{2}$ of the second LPF can be neglected, i.e. $\omega_{1}>>\omega_{2}$, the previous transfer function is rewritten as:

$$
H_{B P}(s) \cong \frac{A_{01} \omega_{1} s}{s^{2}+s \omega_{1}+A_{01} A_{02} \omega_{1} \omega_{2}} .
$$

Comparing to the standard form of the biquad band-pass filter:

$$
H_{B P}(s)=\frac{K_{B P} \frac{\omega_{0}}{Q} s}{s^{2}+s \frac{\omega_{0}}{Q}+\omega_{0}{ }^{2}},
$$

the filter gain $K_{B P}$, the resonant frequency $\omega_{0}$ (central frequency), the quality factor $Q$ and the bandwidth $B W$ of a filter are given by:

$$
\begin{gathered}
K_{B P}=A_{01}, \\
\omega_{0}=\sqrt{\left(1+A_{01} A_{02}\right) \omega_{1} \omega_{2}} \cong \sqrt{A_{01} A_{02} \omega_{1} \omega_{2}}=\sqrt{\frac{g_{m 1}}{C_{1}} \frac{g_{m 2}}{C_{2}}}, \\
Q=\frac{\omega_{0}}{\omega_{1}+\omega_{2}} \cong \frac{\omega_{0}}{\omega_{1}}=\sqrt{\frac{\omega_{2}}{\omega_{1}} A_{01} A_{02}}
\end{gathered}
$$

and

$$
B W \cong f_{1}=\omega_{1} / 2 \pi
$$

respectively. The filter gain and phase characteristics have now the following forms:

$$
\begin{aligned}
\left|H_{B P}(\omega)\right| & =\frac{A_{01} \omega_{1} \omega}{\sqrt{\left(\omega_{1} \omega\right)^{2}+\left(A_{01} A_{02} \omega_{1} \omega_{2}-\omega^{2}\right)^{2}}}, \\
\theta_{B P}(\omega) & =\frac{\pi}{2}-\arctan \frac{\omega_{1} \omega}{A_{01} A_{02} \omega_{1} \omega_{2}-\omega^{2}} .
\end{aligned}
$$

According to (27) we can conclude that at central frequency, $\omega=\omega_{0}$, the phase shift is zero.

During tuning $\omega_{0}$ of a band-pass filter the following condition has to be fulfilled: $K_{B P}$ and $B W$ have to be constant. This can be achieved if $g_{m 1}$ and $\omega_{1}$ are keeping as constants. That implies that the first LPF should be of fixed structure, and $\omega_{0}$ can be adjusted by varying the transconductance $g_{m 2}$, only. 
The phase characteristic of the band-pass filter is defined by (27), where $g_{m 2}$ in $A_{02}=g_{m 2} R_{02}$ is determined, based on (1) and (2), as:

$$
g_{m 2}=g_{m d} \text {. }
$$

The stability of the proposed solution is proved by using the following theorem.

Theorem 2: The phase control loop with the band-pass filter, defined by (27) and (28), with the control (4) and $\theta=\theta_{B P}$, is globally asymptotically stable implying that $\theta_{B P} \rightarrow 0$ for any initial non-zero value of the phase shift.

Proof: The phase loop dynamics is directly obtained by the time-differentiation of (27):

$$
\frac{\mathrm{d} \theta_{B P}}{\mathrm{~d} t}=-\frac{k_{c p} k_{m d} A_{01} R_{02} \omega_{1}^{2} \omega_{2} \omega}{\left(A_{01} A_{02} \omega_{1} \omega_{2}-\omega^{2}\right)^{2}+\left(\omega_{1} \omega\right)^{2}} \theta_{B P},
$$

taking into account (27), (4) and (28). In order to prove that the phase dynamics (29) is stable, we will use the Lyapunov's stability theorem [31]. The Lyapunov's function candidate $E\left(\theta_{B P}\right)$ is chosen to be:

$$
E\left(\theta_{B P}\right)=\frac{1}{2} \theta_{B P}^{2}
$$

As we can see, it is positive definite function $E\left(\theta_{B P}\right)>0$ for $\forall \theta_{B P} \neq 0, E\left(\theta_{B P}\right)=0$ for $\theta_{B P}=0$ and $E\left(\theta_{B P}\right) \rightarrow \infty$ when $\theta_{B P} \rightarrow \infty$. Since its time-derivative:

$$
\frac{\mathrm{d} E\left(\theta_{B P}\right)}{\mathrm{d} t}=-\frac{k_{c p} k_{m d} A_{01} R_{02} \omega_{1}^{2} \omega_{2} \omega}{\left(A_{01} A_{02} \omega_{1} \omega_{2}-\omega^{2}\right)^{2}+\left(\omega_{1} \omega\right)^{2}} \theta_{B P}^{2}<0
$$

is negative definite function for $\forall \theta_{B P} \neq 0$, according to the Lyapunov's stability theorem [31], one can conclude that the equilibrium point of (29) $\theta_{B P}=0$ and, consequently, the overall system are globally asymptotically stable, i.e. the phase $\theta_{B P}$ tends to zero asymptotically from any initial non-zero value.

The dynamical characteristics of the self-tuning $g_{m}-C$ biquad band-pass filter are also verified by the Spice simulation [12]. The IHP design kit for $0.25 \mu \mathrm{m}$ SiGe BiCMOS technology is used [32], as well. The supply voltage $V_{d d}$ is also chosen to be $3 \mathrm{~V}$. Fig. 13 shows time responses of the self-tuning $g_{m}-C$ biquad band-pass filter (power-on sequence). Waveforms of input $V_{\text {in }}$ and output $V_{\text {out }}$ signals are presented in Fig. 13(a). The frequency of the input signal is $f_{i n}=40 \mathrm{MHz}$. The waveforms of UP and DOWN signals, obtained at the outputs of PD, as well as $V_{c t r l}$, are given in Fig. 13(b). The steady-state state is reached in approximately $300 \mathrm{~ns}$. 

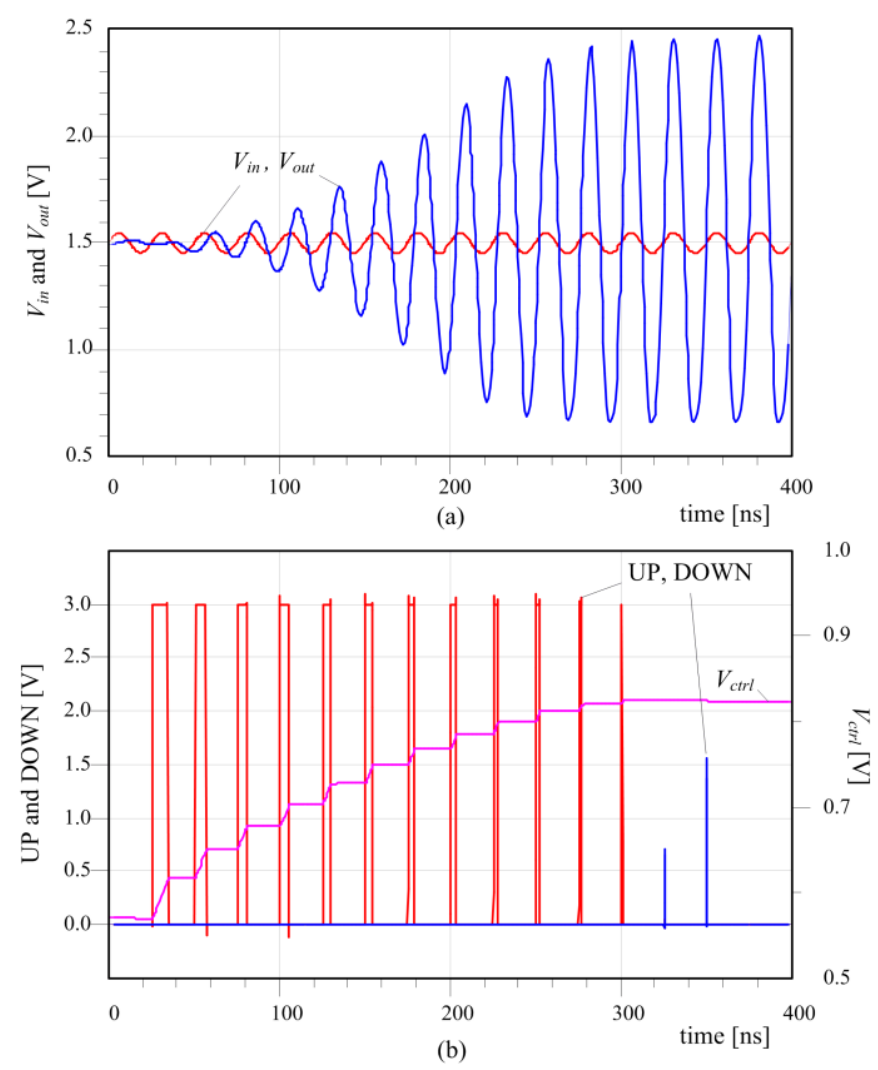

Fig. 13 Time response of self-tuning $g_{m^{-}} C$ biquad band-pass filter : (a) $V_{\text {in }}$ and $V_{\text {out }}$ and (b) UP, DOWN and $V_{c t r l}[12]$

\section{SELF-TUNING LOW-NOISE AMPLIFIER AS A BAND-PASS FILTER}

The block scheme of self-tuning low noise amplifier is presented in Fig. 14 [13]. Lownoise amplifier (LNA) is basically used in the receiving end of the wireless communication system to amplify a very weak received signal to the acceptable level at the specified frequency with adding as little additional noise as possible [33, 34]. Therefore, LNA can be also considered as a band-pass filter.

The LNA schematic is based on the inductively degenerated common source LNA topology [35] and it is shown as a part of block scheme given in Fig. 14. It contains two MOS transistors, $\mathrm{M}_{1}$ and $\mathrm{M}_{2}$, where $\mathrm{M}_{1}$ operate as common-source. The inductor $L_{p r}$ is connected to a DC biasing node. Two active elements, $\mathrm{M}_{1}$ and $\mathrm{M}_{2}$, provide a high LNA gain. 


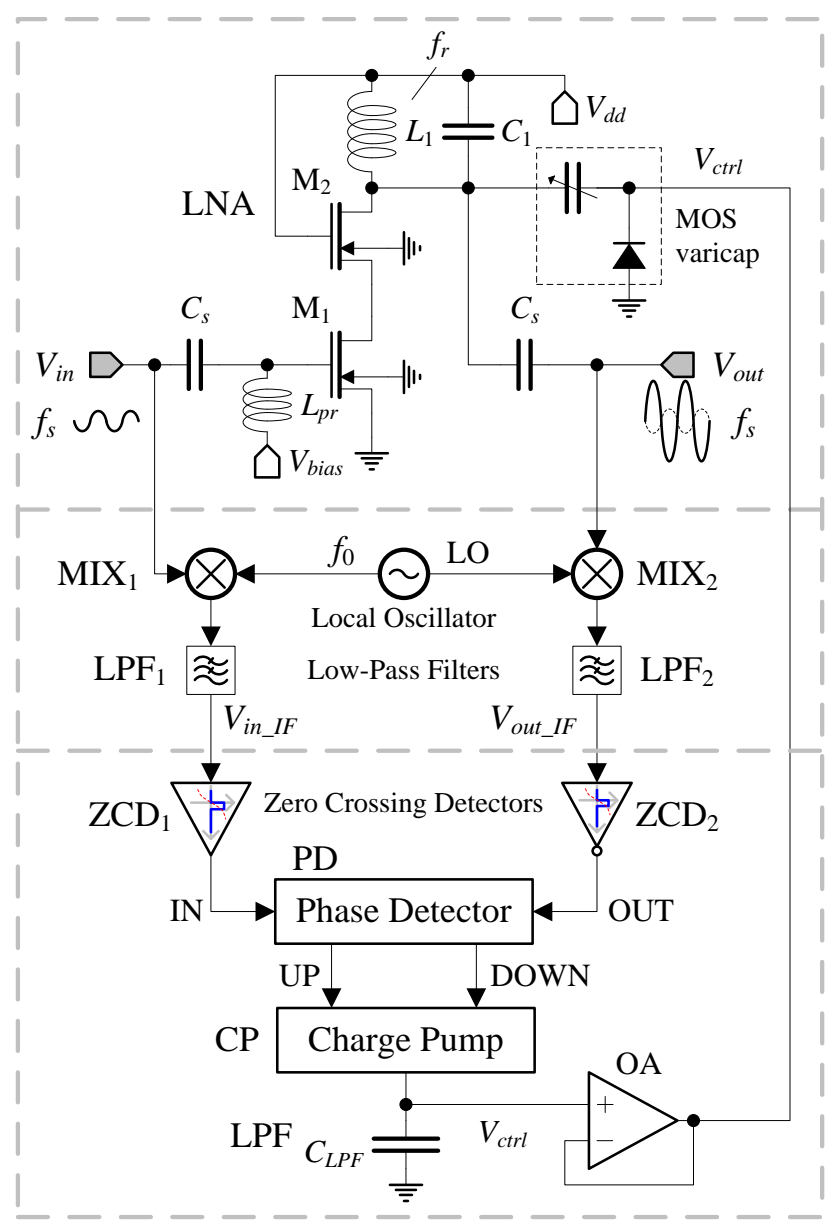

Fig. 14 Block diagram of self-tuning LNA circuit [13]

The output load is implemented as resonant circuit consisting of $L_{1}, C_{1}$ and MVaricap, and the resonant frequency $f_{r}$ is given by

$$
f_{r}=\frac{1}{2 \pi \sqrt{L_{1}\left(C_{1}+C_{\mathrm{MVar}}\right)}} .
$$

The dynamic impedance of resonant circuits, at $f_{r}$, is very high so that the LNA has very high gain, as well. The phase shift of LNA is

$$
\theta_{L N A}=-180^{\circ}-\operatorname{atan}\left(2 Q \frac{f_{s}-f_{r}}{f_{r}}\right),
$$

where $f_{s}$ is a frequency of input signal $V_{i n}$ and $Q$ is a quality factor. 
Elements of a resonant circuit are also chosen in order to provide the high quality factor $Q$ and, consequently, narrow LNA bandwidth $B W=f_{r} / Q$. When $B W$ is narrow then LNA gain is high, the receiver selectivity is good, the attenuation of symmetrical signals in heterodyne receiver is high, and noise level is low.

The operational principle of the phase control loops in the self-tuning LNA is similar to those ones already explained in the previous sections. However, it is very difficult to realize a correct operation of a phase detector at high RF/MW frequency, so the frequency down conversion of signals $V_{\text {in }}$ and $V_{\text {out }}$ should be done by using two mixers $\left(\mathrm{MIX}_{1}, \mathrm{MIX}_{2}\right)$, local oscillator (LO) with frequency $f_{0}$, and two low-pass filters $\left(\mathrm{LPF}_{1}\right.$, $\left.\mathrm{LPF}_{2}\right)$. The results of conversion are signals, $V_{i n \_I F}$ and $V_{\text {out_IF }}$, at lower frequency $f_{0}-f_{s}$. The frequencies $f_{0}+f_{s}$ are eliminated by $\mathrm{LPF}_{1}$ and $\mathrm{LPF}_{2}$. The filtered signals are amplified first and shaped to a rectangular form by zero crossing detectors $\left(\mathrm{ZCD}_{1}, \mathrm{ZCD}_{2}\right)$, composed of CMOS inverter stages. For proper operation of the phase detector (PD), $\mathrm{ZCD}_{2}$ has one inverter stage more than $\mathrm{ZCD}_{1}$, introducing an additional $-180^{\circ}$ phase shift to the output signal, as it is depicted in Fig 15.

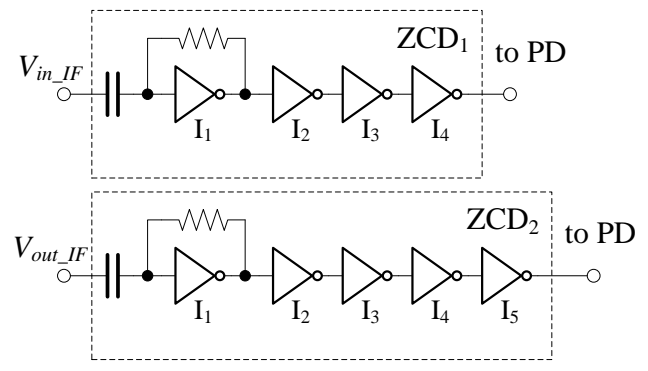

Fig. 15 Zero crossing detector in self-tuning LNA [13]

Finally, the phase error, estimated by PD, is determined by:

$$
\theta_{L N A}^{P D}=-\operatorname{atan}\left(2 Q \frac{f_{s}-f_{r}}{f_{r}}\right) .
$$

The stability of the proposed solution is proved by using the following theorem.

Theorem 3: The phase control loop with LNA, defined by (3), (32) and (34), with the control (4) and $\theta=\theta_{L N A}^{P D}$, is globally asymptotically stable implying that $\theta_{L N A}^{P D} \rightarrow 0$ for any initial non-zero value of the phase shift.

Proof: The phase loop dynamics is directly obtained by time-differentiation of (34):

$$
\frac{d \theta_{L N A}^{P D}}{d t}=-\frac{2 k_{c p} \pi^{2} Q L_{1} C_{M \text { Varicap }}^{3} f_{s} f_{r}^{3}}{V_{\rho}\left(\bar{C}_{\text {MVaricap }}\right)^{2}\left(f_{r}^{2}+4 Q^{2}\left(f_{s}-f_{r}\right)^{2}\right)} \theta_{L N A}^{P D},
$$

taking into account (3), (4) and (34). In order to prove that the phase dynamics (35) is stable, we will use the Lyapunov's stability theorem [31]. The Lyapunov's function candidate $E\left(\theta_{L N A}^{P D}\right)$ is chosen to be: 


$$
E\left(\theta_{L N A}^{P D}\right)=\frac{1}{2}\left(\theta_{L N A}^{P D}\right)^{2}
$$

As we can see, it is positive definite function $E\left(\theta_{L N A}^{P D}\right)>0$ for $\forall \theta_{L N A}^{P D} \neq 0, E\left(\theta_{L N A}^{P D}\right)=0$ for $\theta_{L N A}^{P D}=0$ and $E\left(\theta_{L N A}^{P D}\right) \rightarrow \infty$ when $\theta_{L N A}^{P D} \rightarrow \infty$. Since its time-derivative:

$$
\frac{d E\left(\theta_{L N A}^{P D}\right)}{d t}=-\frac{2 k_{c p} \pi^{2} Q L_{1} C_{\text {MVaricap }}^{3} f_{s} f_{r}^{3}}{V_{\rho}\left(\bar{C}_{\text {MVaricap }}\right)^{2}\left(f_{r}^{2}+4 Q^{2}\left(f_{s}-f_{r}\right)^{2}\right)}\left(\theta_{L N A}^{P D}\right)^{2}<0
$$

is the negative definite function for $\forall \theta_{L N A}^{P D} \neq 0$, according to the Lyapunov's stability theorem [31], one can conclude that the equilibrium point of (35) $\theta_{L N A}^{P D}=0$ and, consequently, the overall system are globally asymptotically stable, i.e. the phase $\theta_{L N A}^{P D} \rightarrow 0$ asymptotically from any initial non-zero value and, consequently $\theta_{L N A} \rightarrow 180^{\circ}$. This means that $V_{\text {in }}$ and $V_{\text {out }}$ signals are synchronized but in an opposite phase, and $V_{\text {out }}$ is significantly amplified.

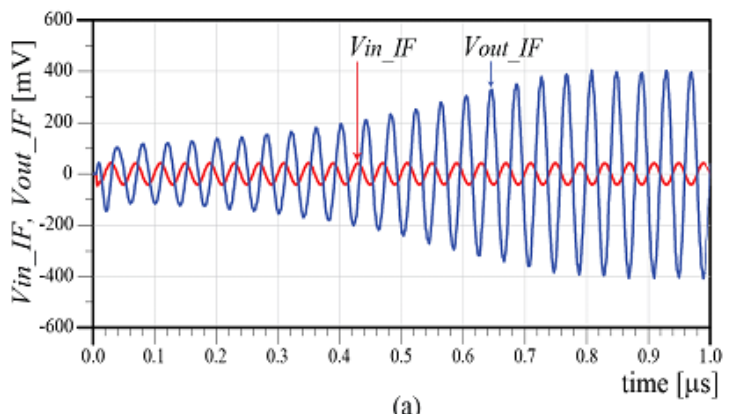

(a)

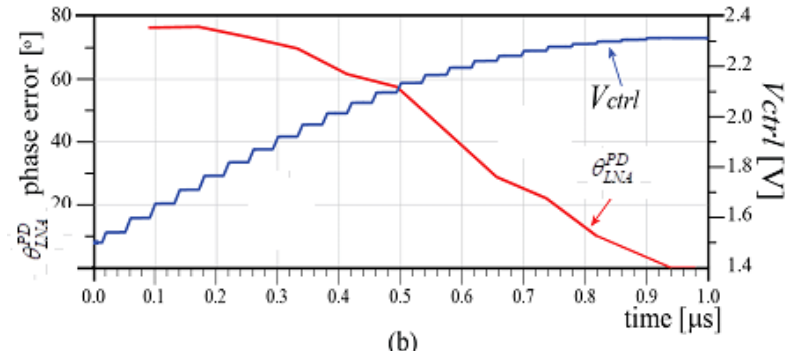

(b)

Fig. 16 Time responses of self-tuning LNA (a) $V_{\text {in_IF }}$ and $V_{\text {out_IF }}$ signals (b) Phase error $\theta_{L N A}^{P D}$ and control voltage $V_{c t r l}[13]$

Verification of the proposed self-tuning LNA is performed by the Spice simulation with the IHP design kit for $0.25 \mu \mathrm{m} \mathrm{SiGe} \mathrm{BiCMOS} \mathrm{[32],} \mathrm{and} \mathrm{supply} \mathrm{voltage} V_{d d}=3 \mathrm{~V}$. Self-tuning LNA has the following characteristics: gain $20 \mathrm{~dB}$, resonant frequency range from 880 up to $950 \mathrm{MHz}, f_{0}=940 \mathrm{MHz}$, bandwidth $B W \in(24.4,26.4) \mathrm{MHz}, Q=36$, noise figure $1.28 \mathrm{~dB}$, and $-1 \mathrm{~dB}$ compression point $-9.8 \mathrm{dBm}$. 
Time responses of the self-tuning LNA for $f_{s}=915 \mathrm{MHz}$ are given in Fig. 16. As it is presented in Fig. 16(a), $V_{\text {in_IF }}$ and $V_{\text {out_IF }}$ signals are synchronized and in opposite phase as expected. We can see from Fig. 16(b) that the steady-state $\left(\theta_{L N A}^{P D}=0\right)$ is reached within $1 \mu \mathrm{s}$. The proposed self-tuning LNA can be also utilized for realization of robust bandpass active filters, based on the master-slave architecture [12] presented in Fig. 17.

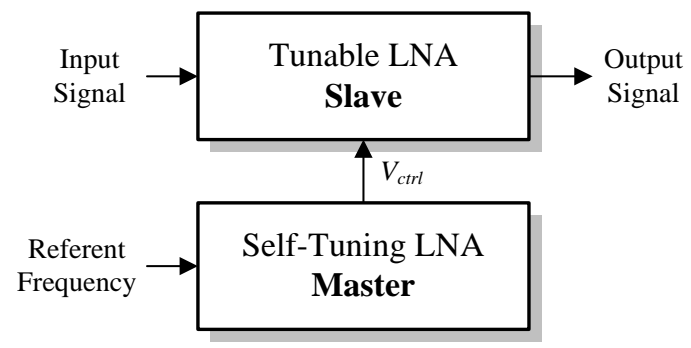

Fig. 17 The block scheme of band-pass filter realized by self-tuning LNA

The structure consists of a master self-tuning LNA and a tunable LNA. The master self-tuning LNA is excited by the referent frequency source $f_{s}$ and it generates control voltage, $V_{c t r l}$. The slave LNA is of an identical structure as one in the master. It is driven with the same control voltage $V_{c t r l}$, generated by the master self-tuning LNA. The slave is used for filtering and amplification of the input signal.

\section{SELF-TUNING LOW-PASS FILTER}

The structure of phase control loop with band-pass filter can be modified to be used as a self-tuning low-pass filter, if the $V_{L P}$ output of filter, presented in Fig. 8, is taken as an output. Since it is difficult to generate the reference signal with the constant phase shift of $-90^{\circ}$ and with the same frequency as an input signal, the feedback is closed via signal $V_{B P}$ as in the previous case. That is why the stability analysis of phase control is the same as in the Section 5. As the $V_{L P}$ output of filter is used, the input signal is passed through the low pass filter, whose transfer function is:

$$
H_{L P}(s)=\frac{V_{L P}}{V_{\text {in }}}=\frac{A_{1}(s) \cdot A_{2}(s)}{1+A_{1}(s) \cdot A_{2}(s)}=\frac{\omega_{1} \cdot \omega_{2} \cdot A_{01} \cdot A_{02}}{s^{2}+s\left(\omega_{1}+\omega_{2}\right)+\left(1+A_{01} \cdot A_{02}\right) \cdot \omega_{1} \cdot \omega_{2}} .
$$

Assuming that $A_{01}, A_{02} \gg 1,(38)$ is rewritten as:

$$
H_{L P}(s) \cong \frac{\omega_{1} \cdot \omega_{2} \cdot A_{01} \cdot A_{02}}{s^{2}+s\left(\omega_{1}+\omega_{2}\right)+A_{01} A_{02} \omega_{1} \omega_{2}}
$$

Comparing to the standard form of a biquad low-pass filter:

$$
H_{L P}(s)=\frac{\omega_{c}{ }^{2}}{s^{2}+s \frac{\omega_{c}}{Q}+\omega_{c}{ }^{2}},
$$


the filter cut-off frequency $\omega_{c}$ is almost equal to the central frequency of a band-pass filter:

$$
\omega_{c}=\omega_{0}
$$

and the quality factor $Q$ of a filter are given by:

$$
Q=\frac{\omega_{c}}{\omega_{1}+\omega_{2}} \cong \frac{\sqrt{\omega_{1} \cdot \omega_{2} \cdot A_{01} \cdot A_{02}}}{\omega_{1}+\omega_{2}} .
$$

The filter gain and phase characteristics are:

$$
\begin{gathered}
\left|H_{L P}(\omega)\right|=\frac{\omega_{1} \omega_{2} A_{01} A_{02}}{\sqrt{\left(\omega_{1}+\omega_{1}\right)^{2} \cdot \omega^{2}+\left(\left(1+A_{01} A_{02} \omega_{1} \omega_{2}\right)-\omega^{2}\right)^{2}}}, \\
\theta_{L P}(\omega)=-\arctan \frac{\left(\omega_{1}+\omega_{2}\right) \cdot \omega}{\left(1+A_{01} A_{02}\right) \omega_{1} \omega_{2}-\omega^{2}}
\end{gathered}
$$

According to (44) we can conclude that at cut-off frequency, $\omega=\omega_{c}$, the phase shift is $-90^{\circ}$. This is illustrated in Fig. 18, where comparative presentation of band-pass and lowpass gain and phase characteristics are given.
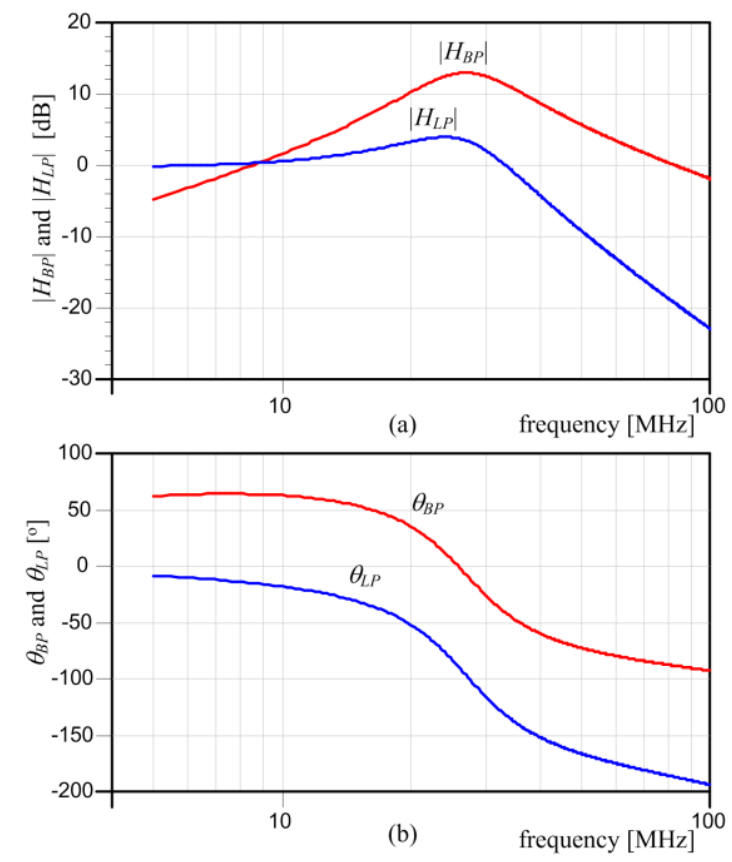

Fig. 18 Gain and phase characteristics of band-pass and low-pass filter 
Fig. 19 depicts time responses of self-tuning band-pass and low-pass filters. Waveforms of input signal $V_{i n}$, band-pass filter output signal $V_{B P}$, and low-pass filter output signal $V_{L P}$ are given in Fig. 19. As it is expected, signal $V_{L P}$ is phase shifted for $-90^{\circ}$ and amplified in comparison to the input signal $V_{i n}$.

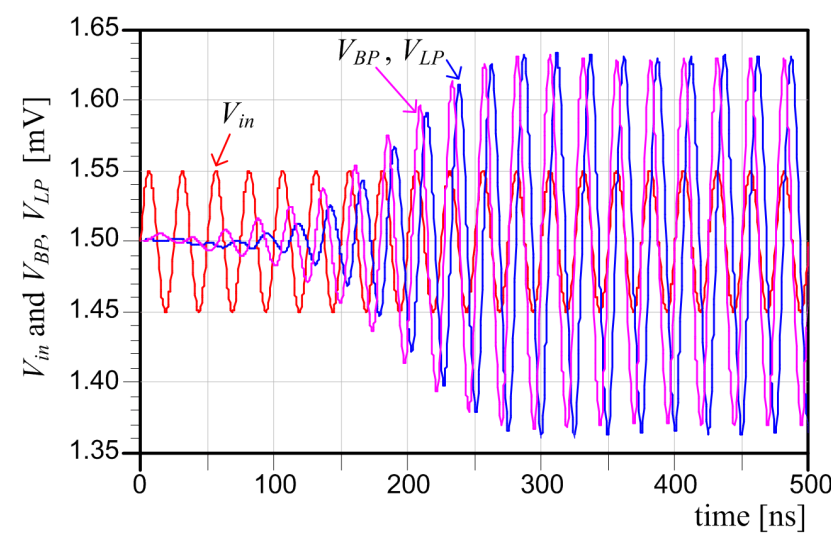

Fig. 19 Time response of self-tuning low-pass filter

As we can see, the cut-off frequency of a low-pass filter in the proposed phase control loop could be adjusted, and it is closed to the frequency of the input signal. Having this in mind, it is possible to use this low-pass filter as an RF channel selector.

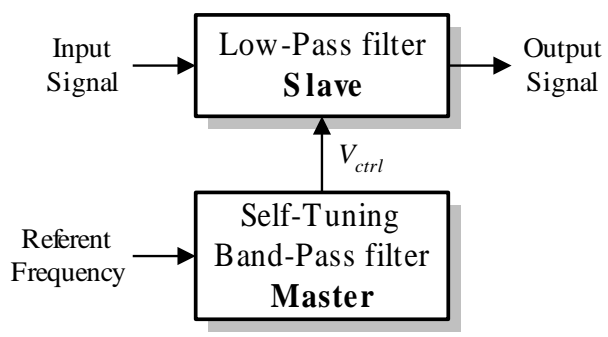

Fig. 20 The block scheme of channel selector

Fig. 20 presents the low-pass filter application as an RF channel selector. There are two filters - master and slave. The master filter is based on the phase control loop with a band-pass filter and it is excited with a referent frequency source that determines the cutoff frequency of the low-pass filter. It also generates the control voltage, $V_{c t r l}$, which drives the slave filter of the identical structure as the master band-pass one, but where the $V_{L P}$ is used as the filter output. In that way, the RF channels are chosen by changing the frequency of the referent signal source. 


\section{SELF-TUNING NOTCH FILTER}

The operational principle of self-tuning notch filter is also based on a previously explained phase control loop strategy [14]. The block diagram of this self-tuning filter as well as its main component, a voltage-controlled notch filter (VCNF), are shown in Fig. 21. In order to provide imaginary zeros in the notch filter (NF) transfer function, NF is realized as a voltage divider, composed of a resistor, realized by an OTA with transconductance $g_{m}$, and a series resonant circuit composed of a capacitor $C_{1}$ and a positive impedance inverter (Gyrator), implemented by using $\mathrm{OTA}_{1}, \mathrm{OTA}_{2}$ and a capacitor $C_{2}$.

The NF transfer function is:

$$
H_{N F}(s)=\frac{V_{N F}(s)}{V_{i n}(s)}=\frac{s^{2}+\frac{g_{m 1} g_{m 2}}{C_{1} C_{2}}}{s^{2}+s \frac{C_{1}}{g_{m}} \frac{g_{m 1} g_{m 2}}{C_{1} C_{2}}+\frac{g_{m 1} g_{m 2}}{C_{1} C_{2}}} .
$$

Comparing the standard form of the NF transfer function:

$$
H_{N F}(s)=\frac{s^{2}+\omega_{0}^{2}}{s^{2}+s \frac{\omega_{0}}{Q}+\omega_{0}^{2}}
$$

with (45), yields:

$$
\begin{gathered}
\omega_{0}=\sqrt{\frac{g_{m 1} g_{m 2}}{C_{1} C_{2}}}, \\
Q=\frac{g_{m}}{C_{1} \omega_{0}}=g_{m} \sqrt{\frac{1}{g_{m 1} g_{m 2}} \frac{C_{2}}{C_{1}}},
\end{gathered}
$$

for the central frequency $\omega_{0}$ and the quality factor $Q$. The NF phase characteristic is given by:

$$
\theta_{N F}(\omega)=-\operatorname{atan} \frac{\frac{\omega_{0}}{Q} \omega}{\omega_{0}^{2}-\omega^{2}}
$$

where $\theta_{N F}\left(\omega_{0}\right)=-180^{\circ}$.

The VCNF circuit presented in Fig. 21 acts both as the NF, where the output $V_{N F}$ is used, as well as the band-pass filter (BF), where $V_{B F}$ is considered as an output signal. The $\mathrm{BF}$ transfer function is given by:

$$
H_{B F}(s)=\frac{V_{B F}(s)}{V_{i n}(s)}=\frac{s \frac{g_{m 1}}{C_{2}}}{s^{2}+s \frac{C_{1}}{g_{m}} \frac{g_{m 1} g_{m 2}}{C_{1} C_{2}}+\frac{g_{m 1} g_{m 2}}{C_{1} C_{2}}} .
$$




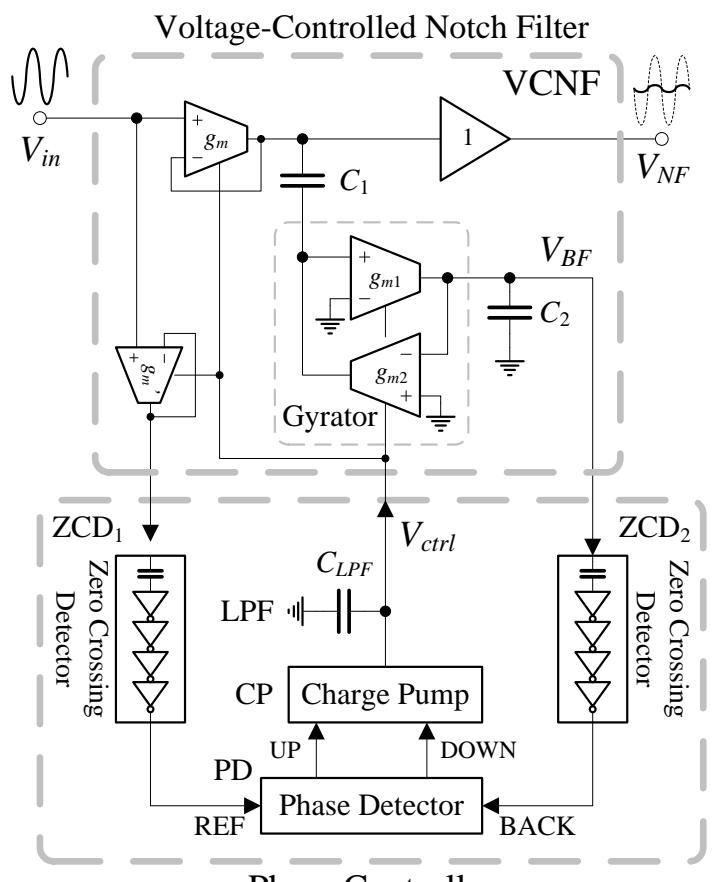

Phase Controller

Fig. 21 Self-tuning notch filter [14]

As we can see, the central frequency $\omega_{0}$ and the quality factor $Q$ of both $\mathrm{NF}$ and $\mathrm{BF}$ filters have identical values. The BF phase characteristic is described by:

$$
\theta_{B F}(\omega)=\frac{\pi}{2}-\operatorname{atan} \frac{\frac{\omega_{0}}{Q} \omega}{\omega_{0}^{2}-\omega^{2}}
$$

and $\theta_{B F}\left(\omega_{0}\right)=0$.

At the center frequency $\omega_{0}$, the amplitude of NF output signal is very low, and consequently, the signal/noise ratio is also low. Therefore, we use the BF output to close the phase control loop, since it has maximum amplitude at $\omega_{0}$, thanks to the fact that the $\mathrm{BF}$ acts as a selective amplifier of the input signal, as well [12]. This approach is justified from two reasons. First, the center frequency $\omega_{0}(47)$ and the quality factor $Q(48)$ of NF and BF are identical and, therefore, these filter characteristics change with PVT variations in the same manner. Second, the phase-shift of a well-tuned BF is equal to zero, and the realization of the phase control loop does not need additional phase correction of the output feedback signal $V_{B F}$.

The control voltage, $V_{c t r l}$ defines the values of OTA transconductances $g_{m}, g_{m}{ }^{\prime}, g_{m 1}$, $g_{m 2}$ in VCNF according to

$$
g_{m}=k_{m}\left(V_{c t r l}-V_{T h}\right), g_{m}^{\prime}=k_{m}^{\prime}\left(V_{c t r l}-V_{T h}\right), g_{m_{i}}=k_{m_{i}}\left(V_{c t r l}-V_{T h}\right), i=1,2 .
$$


If OTA, OTA 1 and OTA 2 are identical, realized by replicating the electrical schemes, the variations of $g_{m}, g_{m 1}$ and $g_{m 2}$ under the influence of the control voltage $V_{c t r l}$ are the same. In this case, the quality factor $Q$, defined by (48), can be expressed as

$$
Q=\sqrt{\frac{C_{2}}{C_{1}}} .
$$

The stability proof of the phase control loop is given in the following theorem.

Theorem 4: The phase loop, described by (47), (48), (51) and (52), with the control (4), and $\theta=\theta_{B F}$, is globally asymptotically stable, i.e. $\theta_{B F} \rightarrow 0$ asymptotically for any initial non-zero value of the phase-shift.

Proof: The phase loop dynamics can be directly derived by time-differentiation of (51):

$$
\frac{\mathrm{d} \theta_{B F}}{\mathrm{~d} t}=-\frac{k_{m} k_{c p} \omega\left(\omega_{0}^{2}+\omega^{2}\right)}{C_{2}\left(\omega_{0}^{2}-\omega^{2}\right)^{2}+C_{1}\left(\omega \omega_{0}\right)^{2}} \theta_{B F},
$$

taking into account (47), (48), (52) (53), $g_{m}=g_{m 1}=g_{m 2}$ and $k_{m}=k_{m 1}=k_{m 2}$.

We will use the Lyapunov's stability theorem [31] to prove the stability of the proposed self-tuning NF. The Lyapunov's function candidate $E\left(\theta_{B F}\right)$ is selected as:

$$
E\left(\theta_{B F}\right)=\frac{1}{2} \theta_{B F}^{2}
$$

that is a positive definite function i.e. $E\left(\theta_{B F}\right)>0$ for $\forall \theta_{B F} \neq 0, E\left(\theta_{B F}\right)=0$ for $\theta_{B F}=0$ and $E\left(\theta_{B F}\right) \rightarrow \infty$ when $\theta_{B F} \rightarrow \infty$. Since its time-derivative:

$$
\frac{\mathrm{d} E\left(\theta_{B F}\right)}{\mathrm{d} t}=-\frac{k_{m} k_{c p} \omega\left(\omega_{0}^{2}+\omega^{2}\right)}{C_{2}\left(\omega_{0}^{2}-\omega^{2}\right)^{2}+C_{1}\left(\omega \omega_{0}\right)^{2}} \theta_{B F}^{2}<0
$$

is a negative definite function for $\forall \theta_{B F} \neq 0$, we conclude that the equilibrium point of (54) $\theta_{B F}=0$ and, consequently, the overall self-tuning NF are globally asymptotically stable, i.e. that the phase $\theta_{B F}$ asymptotically tends to zero from any initial non-zero value.

The self-tuning OTA-C NF is implemented in $0.13 \mu \mathrm{m}$ SiGe BiCMOS technology [36] with $1.8 \mathrm{~V}$ supply voltage, and power consumption less than $3.6 \mathrm{~mW}$. The charging (discharging) current of the $\mathrm{CP}$ is $I_{C P}=60 \mu \mathrm{A}$, and the low-pass filter capacitance is $C_{L P F}$ $=4 \mathrm{pF}$.

Time responses of the self-tuning NF with phase control loop are depicted in Fig. 22. The waveforms of $V_{i n}, V_{N F}$ and $V_{B F}$, are presented in Fig. 22(a). The frequency of input signal is $f=100 \mathrm{MHz}$ and it corresponds to the desired filter center frequency $f_{0}$. In Fig. 22(b), the waveforms of UP and DOWN signals, generated by PD, as well as $V_{c t r l}$, are given. The steady-state is reached after $100 \mathrm{~ns}\left(10 / f_{0}\right)$. The phase control loop is locked then, the phases of signals $V_{i n}$ and $V_{B F}$ are equal, the center frequency is identical to the one of the input signal, and the maximal attenuation of the proposed NF is achieved. 

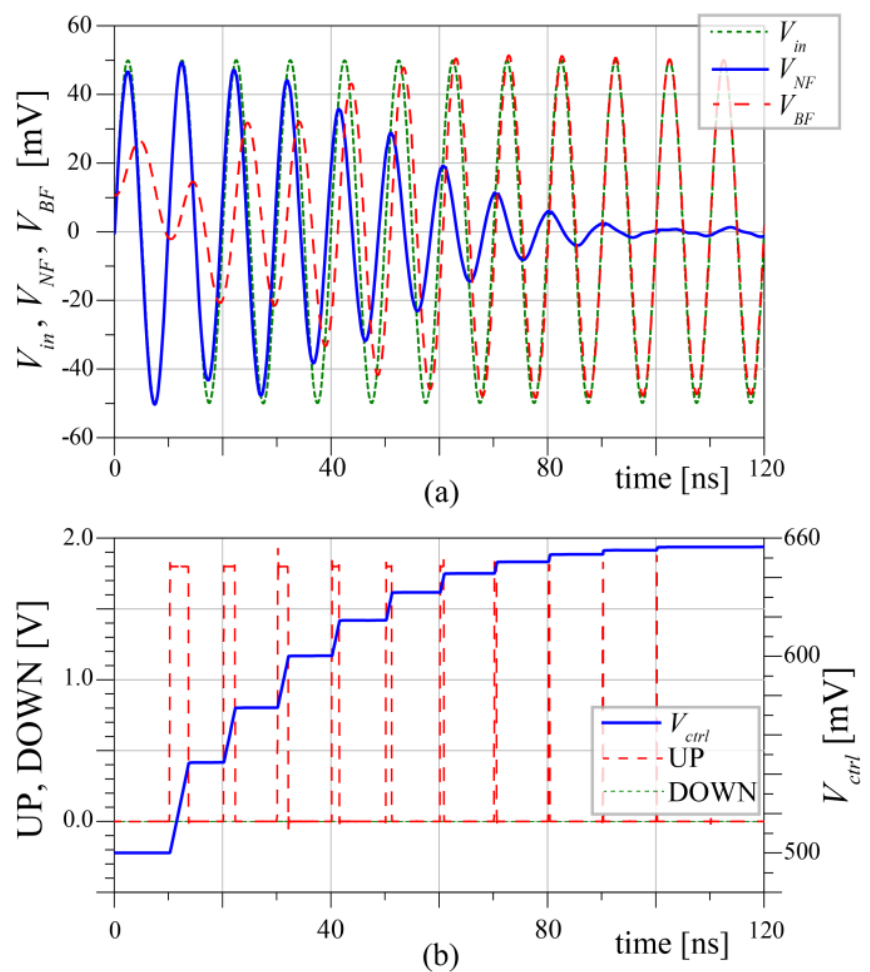

Fig. 22 Time response of self-tuning notch filter: (a) $V_{i n}, V_{N F}$ and $V_{B F}$ and (b) UP, DOWN and $V_{c t r l}[14]$

\section{CONCLUSION}

This paper deals with the synthesis of phase control loops based on voltage-controlled active filters [11-14] resulting in self-tuning all-pass, band-pass, low-pass and notch filters. The crucial property of the proposed approach relates to the phase correction of an analogue input signal, which is achieved by varying the delay of a signal, passing through the filter. In that way, the filter phase characteristics are tuned by controlling the transconductance value $g_{m}$ of operational transconductance amplifier (OTA) or the capacitance of MOS varicap element. The control is realized in the manner similar to the operational principle of a delay locked loop in digital domain, which is a new approach in the design of phase control loops. The stability of phase control loops is analyzed by using the Lyapunov's control method [31]. Digital simulation results of system dynamics after power on (the worst case operation) are also presented for all four cases. It is shown that the proposals provide good properties such as the first-order loops, high stability and short settling times, i.e. low latencies resulting in high system throughputs. Owing to small dimensions of filter constituents, these structures are suitable for implementation in VLSI CMOS technology. 
Acknowledgement: This work has been supported by the Ministry of Education, Science and Technological Development of the Republic of Serbia.

\section{REFERENCES}

[1] F. Dülger, E. Sánchez-Sinencio, J. Silva-Martínez, "A 1.3-V 5-mW fully integrated tunable bandpass filter at $2.1 \mathrm{GHz}$ in 0.35- $\mu \mathrm{m}$ CMOS", IEEE J. Solid-State Circuits, vol. 38, no. 6, pp. 918-928, 2003. [Online]. Available: http://dx.doi.org/10.1109/JSSC.2003.811867

[2] Gh. Z Fatin, Z. D. K Kanani, "Very low power band-pass filter for low-IF applications", J. Circuits, Systems and Computers, vol. 17, no. 4, pp. 685-701, 2008. [Online]. Available: http://dx.doi.org/10.1142/ S0218126608004496

[3] D. Chamia, A. Kaiser, A. Cathelin, D. Belot, "A gm-C low-pass filter for zero-IF mobile applications with a very wide tuning range", IEEE J. Solid-State Circuits, vol. 40, no. 7, pp. 1443-1450, 2005. [Online]. Available: http://dx.doi.org/10.1109/JSSC.2005.847274

[4] H. Yamazaki, K. Oishi, K. Gotoh, "An accurate center frequency tuning scheme for 450-kHz CMOS gm-C bandpass filters", IEEE J. Solid-State Circuits, vol. 34, no. 12, pp. 1691-1697, 1999. [Online]. Available: http://dx.doi.org/10.1109/4.808894

[5] H. Voorman, H. Veenstra, "Tunable high-frequency gm-C filters", IEEE J. Solid-State Circuits, vol. 35, no. 8, pp. 1097-1108, 2000. [Online]. Available: http://dx.doi.org/10.1109/4.859498

[6] S. Solís-Bustos, J. Silva-Martínez, F. Maloberti, E. Sánchez-Sinencio, "A 60-dB dynamic-range CMOS sixth-order 2.4-Hz low-pass filter for medical applications", IEEE Trans. CAS - II, vol. 47, no. 12, pp. 1391-1398, 2000. [Online]. Available: http://dx.doi.org/10.1109/82.899631

[7] X. Qian, Y. P. Xu, X. Li, “A CMOS continuous-time low-pass notch filter for EEG Systems”, Analog Integrated Circuits and Signal Processing, vol. 44, no. 3, pp. 231-238, 2005. [Online]. Available: https://doi.org/10.1007/s10470-005-3007-x

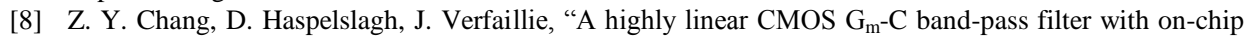
frequency tuning", IEEE J. Solid-State Circuits, vol. 32, no. 3, pp. 388-397, 1997. [Online]. Available: http://dx.doi.org/10.1109/4.557637

[9] R. Dobkin, R. Ginosar, "Fast universal synchronizers", Integrated Circuit and System Design. Power and Timing Modeling, Optimization and Simulation, PATMOS 2008, Lisbon, Portugal, September 1012, 2008. Revised Selected Papers, L. Svensson and J. Monteiro, Eds., Lecture Notes in Computer Science, vol 5349. Springer, Berlin, Heidelbergvol. 5349, pp. 199-208, Springer, 2009. [Online]. Available: https://doi.org/10.1007/978-3-540-95948-9_20

[10] J. W. M. Rogers, C. Plett, "A 5-GHz radio front-end with automatically Q-tuned notch filter and VCO", IEEE J. Solid-State Circuits, vol. 38, no. 9, pp. 1547-1554, 2003. [Online]. Available: http://dx.doi.org/10.1109/ JSSC.2003.815915

[11] G. S. Jovanović, D. B. Mitić, M. K. Stojčev, D. S. Antić,"Phase-synchronizer based on gm-C all-pass filter chain", Advances in Electrical and Computer Engineering, vol. 12, no. 1, pp. 39-44, 2012. [Online]. Available: http://dx.doi.org/10.4316/AECE.2012.01007

[12] G. S. Jovanović, D. B. Mitić, M. K. Stojčev, D. S. Antić, "Self-tuning biquad band-pass filter", J. Circuits, Systems and Computers, vol. 22, no. 3, pp. 1-19, 2013. [Online]. Available: http://dx.doi.org/10.1142/ S0218126613500084

[13] G. S. Jovanović, D. B. Mitić, M. K. Stojčev, D. S. Antić, "Self-tuning low-noise amplifier", Facta Universitatis, Series: Automatic Control and Robotics, vol. 12, no. 2, pp. 139-145, 2013. [Online]. Available: http://casopisi.junis.ni.ac.rs/index.php/FUAutContRob/article/view/52

[14] G. S. Jovanović, D. B. Mitić, M. K. Stojčev, D. S. Antić, "Self-tuning OTA-C notch filter with constant Q-factor", J. of Circuits, Systems and Computers, vol. 25, no. 5, 2016. [Online]. Available: http://dx.doi.org/10.1142/S0218126616500456

[15] C. Yoo, S.-W. Lee, W. Kim, "A $\pm 1.5-\mathrm{V}, 4-\mathrm{MHz}$ CMOS continuous-time filter with a single-integrator based tuning", IEEE J. Solid-State Circuits, vol. 33, no. 1, pp. 18-27, 1998. [Online]. Available: http://dx.doi.org/ $10.1109 / 4.654933$

[16] J. Maneatis, "Low-jitter process-independent DLL and PLL based on self-biased techniques", IEEE J. SolidState Circuits, vol. 31, no. 11, pp. 1723-1732, 1996. [Online]. Available: http://dx.doi.org/10.1109/JSSC. 1996.542317 
[17] Y. Moon, J. Choi, K. Lee, D.-K. Jeong and M.-K. Kim, "An all-analog multiphase DLL using a replica delay line for wide-range operation and low-jitter performance", IEEE J. Solid-State Circuits, vol. 35, no. 3, pp. 377-384, 2000. [Online]. Available: http://dx.doi.org/10.1109/4.826820

[18] M. K. Stojčev, G. S. Jovanović, "Clock aligner based on delay-locked loop with double edge synchronization", Microelectronics Reliability, vol. 48, no. 1, pp. 158-166, 2008. [Online]. Available: http://dx.doi.org/10.1016/j.microrel.2007.02.025

[19] M.-J. E. Lee, W. J. Dally, T. Greer, H.-T. Ng, R. Farjad-Raad, J. Poulton, R. Senthinathan, "Jitter transfer characteristics of delay-locked loops - Theories and design techniques", IEEE J. of Solid State Circuits, vol. 38, no. 4, pp. 614-621, 2003. [Online]. Available: http://dx.doi.org/10.1109/JSSC.2003.809519

[20] P.-H. Yang, J.-S. Wang, "Low-voltage pulse width control loops for SOC applications", IEEE J. of Solid State Circuits, vol. 37, no. 10, pp. 1348-1351, 2002. [Online]. Available: 10.1109/JSSC.2002.803050

[21] G. S. Jovanović, M. K. Stojčev, "A delay locked loop for analog signal", in Proceedings of $9^{\text {th }}$ International Conference TELSIKS, Niš, Serbia, 7-9 October 2009vol. 1, pp. 233-236, 2009. [Online]. Available: http://es.elfak.ni.ac.rs/Papers/TELSIKS2009_JovanovicStojcev.pdf

[22] Y. Özcelep, A. Kuntman, H. Kuntman, "On the degradation of OTA-C based CMOS low-power filter circuits for biomedical instrumentation", TJEECS: Turkish Journal of Electrical Engineering \& Computer Sciences, vol. 20, no. 2, pp. 1359-1368, 2012. [Online]. Available: (DOI: 10.3906/elk-1106-4

[23] G. Düzenli, Y. Kiliç, H. Huntman, A. Ataman, "On the design of low-frequency filters using CMOS OTAs operating in subtreshhold region" Microelectronics Journal, vol. 30, no. 1, pp. 45-54. 1999. [Online]. Available: https://doi.org/10.1016/S0026-2692(98)00084-6

[24] F. Maloberi, Analog Design for CMOS VLSI Systems, Kluwer Academic Publisher, Boston, USA, 2001.

[25] R.G. Bozomitu and N. Cojan, "A VLSI implementation of a new low voltage 5th order differential $\mathrm{g}_{\mathrm{m}}-\mathrm{C}$ lowpass filter with auto-tuning loop in CMOS technology", Advances in Electrical and Computer Engineering, vol. 11, no. 1, pp. 23-30, 2011. [Online]. Available: http://dx.doi.org/10.4316/AECE.2011.01004

[26] T. S. Rodriguez, C. I. Lujan-Martinez, R. G. Carvajal, J. Ramirez-Angulo, A. Lopez-Martin, “A CMOS linear tunable transconductor for continuous-time tunable $\mathrm{g}_{\mathrm{m}}-\mathrm{C}$ filters", in Proceedings of IEEE International Symposium on Circuits and Systems (ISCAS), 18-21 May 2008, Sheraton Seattle Hotel, Seattle, Washington, USA, pp. 912-915, 2008. [Online]. Available: https://doi.org/10.1109/ISCAS.2008.4541567

[27] M. Pedro, J. Galán, T. Sánchez-Rodríguez, F. Muñoz, R. G. Carvajal, A. López-Martin, ”A low-pass filter with automatic frequency tuning for a bluetooth receiver", in Proceedings of $17^{\text {th }}$ IEEE International Conference on Electronics, Circuits, and Systems (ICECS), Athens, Greece, 12-15 December 2010, pp. 462-465, 2008. [Online]. Available: https://doi.org/10.1109/ICECS.2010.5724549

[28] D. J. Cassan, J. R. Long, "A 1-V transformer-feedback low-noise amplifier for 5-GHz wireless LAN in

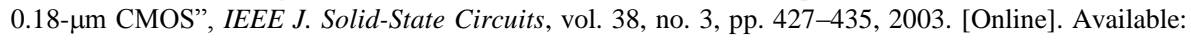
http://dx.doi.org/10.1109/JSSC.2002.808284

[29] B. P. Das, N. Watson, L. Yonghe, "Wide tunable all pass filter using OTA as active component," International Conference on Signals and Electronic Systems (ICSES), pp. 379-382, 2010.

[30] C.-M. Chang, B.M. Al-Hashimi, "Analytical synthesis of voltage mode OTA-C all-pass filters for high frequency operation, circuits and systems", in Proceedings of IEEE International Symposium on Circuits and Systems (ISCAS), Bangkok, Thailand, May 25-28, 2003, vol. 1, pp. 461-464, May 2003. [Online]. Available: http://dx.doi.org/ 10.1109/ISCAS.2003.1205600

[31] H. K. Khalil, Nonlinear Systems. Upper Saddle River NJ: Prentice Hall, 1996

[32] IHP-Microelectronics, SiGe:C BiCMOS technologies for MPW \& prototyping. [Online] Cited 2013-08-30. Available at: http://www.ihp-microelectronics.com/en/services/mpw-prototyping/sigec-bicmos-technologies.html

[33] D. Gomez, M. Sroka, J. L. Gonzalez Jimenez, "Process and temperature compensation for RF low-noise amplifiers and mixers", IEEE Transactions on Circuits and Systems I, vol. 57, no. 6, pp. 1204-1211, 2010. [Online]. Available: http://dx.doi.org/10.1109/TCSI.2009.2031707

[34] T Das, A. Gopalan, C Washburn, P. R. Mukund, "Self-calibration of input-match in RF front-end circuitry", IEEE Transactions on Circuits and Systems II, vol. 52, no. 12, pp. 821-825, 2005. [Online]. Available: http://dx.doi.org/10.1109/TCSII.2005.853893

[35] D. K. Shaeffer, T. H. Lee, “A 1.5-V, 1.5-GHz CMOS low noise amplifier”, IEEE J. Solid-State Circuit, vol. 32, no. 5, pp. 745-759, 1997. [Online]. Available: http://dx.doi.org/10.1109/4.568846

[36] IHP-Microelectronics, SiGe:C BiCMOS Technologies for MPW \& Prototyping, [Online] Cited 2015 , Available at: http://www.ihp-microelectronics.com/en/services/mpw-prototyping/sigec-bicmos-technologies.html 